\title{
Detection and significance of subclinical mitral regurgitation by colour Doppler techniques
}

Two papers in this issue demonstrate clinical consequences from the presence of clinically silent mitral regurgitation in paediatric and adolescent populations. Both are potentially important as the development of progressive doxorubicin cardiomyopathy $^{1}$ and the presence of rheumatic carditis ${ }^{2}$ have long term implications. A parallel example, which highlights some of the problems inherent in attempting to derive clinical conclusions from the presence of mild regurgitation in an adult population, exists in the recent "phen/fen" controversy. ${ }^{3}$ Attempting to extract useful prognostic information from the presence of clinically silent, Doppler detected, mitral regurgitation through morphologically normal valves is not new-its negative impact on the prognosis of acute infarction has been recognised for over a decade. ${ }^{4}$ However, a fundamental prerequisite in attempting to utilise this type of information is an appreciation of the factors which underlie the presence of the colour flow signal. The appearance of mitral regurgitation is dependent upon the mechanical substrate for regurgitation itself, the fluid dynamics of the resultant flow disturbance, and lastly, processing using a colour flow mapping algorithm to produce an image. In particular, we need to question the reproducibility of findings obtained by different examiners and between different echo system implementations of colour flow mapping before we can feel comfortable about extrapolating these findings into clinical practice.

\section{Mechanical substrates for regurgitation}

In some very mild cases of rheumatic carditis and subtle forms of the "phen/fen" effect, leaflet damage not apparent on two dimensional echo is the intuitive mechanism of subclinical regurgitation. However, in the majority of cases, the valve is merely responding to changes in ventricular geometry, pressure loading, or both. In a series of elegant studies, ${ }^{5}$ Levine's group has demonstrated that functional mitral regurgitation is a dynamic entity, with maximal flow occurring in early and late systole accompanied by a characteristic mid-systolic dip, ${ }^{6}$ suggesting a role for the transmitral systolic pressure gradient in determining the severity of regurgitation. ${ }^{7}$ In contrast to the study of Allen and colleagues, ${ }^{1}$ three dimensional studies in animal models ${ }^{8}$ and two dimensional clinical observations, ${ }^{6}$ suggest that depressed systolic function in isolation does not result in mitral regurgitation in the absence of ventricular dilatation. The principal requirements for functional mitral regurgitation include outward papillary muscle displacement, annular dilatation, and possibly a reduction in leaflet closing forces. $^{5}$

\section{Physical properties of regurgitant jet sprays}

Despite the presence of ambient counterflows in the atrium, mitral regurgitation behaves as a turbulent free jet. $^{9-11}$ When flow accelerates towards a restrictive regurgitant orifice, the flow field progressively narrows in proportion to the relative increase in velocity until it passes through the orifice (fig 1). After this, the laminar core con- tinues to focus, reaching its narrowest point (the vena contracta) just inside the left atrium. While accelerating through the isovelocity shells preceding the mitral valve and once within the vena contracta itself, flow is essentially laminar, providing the opportunity for relatively precise quantification of flow in experimental studies. ${ }^{12}{ }^{13}$ Beyond this point there is no further acceleration and vortices develop as the kinetic energy of the laminar core is progressively dissipated by incorporation of mass from the surrounding atrial blood. ${ }^{14}$ This region is termed the jet intrusion core or flow development region and typically spans five orifice diameters. The area beyond the jet intrusion zone is termed the fully developed zone and the combination of these two regions produces a characteristic turbulent deceleration zone around and beyond the intrusion core. The jet core (or intrusion distance) varies as a function of velocity $\times$ orifice diameter and thus with larger orifices, a greater flow volume will be required to yield a high enough velocity for an equivalently long intrusion distance. Importantly, as the generation of the fully developed core is a function of kinetic energy (pressure) and not simply volume, elevations of ventricular systolic pressure produce larger jet sprays in the absence of any increase in orifice area. ${ }^{15}$ Other factors influencing the transformation of the jet core into the fully developed turbulent jet spray region include atrial pressure, pulmonary venous flow, and contact with the atrial walls.

\section{Effect of instrument settings on flow visualisation}

The fundamental, and well recognised, problem is that colour flow imaging is a misnomer as it provides information on the spatial distribution of velocity but not flow itself. Flow is accessible (velocity $\times$ area $=$ flow rate), but as we do not currently have easy access to regurgitant orifice measurements in clinical practice, we are tempted into ignoring area and relating velocity fields directly to flow. The next problem is that conventional colour Doppler techniques for the detection of regurgitant flows rely predominantly on the magnitude of the kinetically determined turbulent jet spray deceleration zone and not

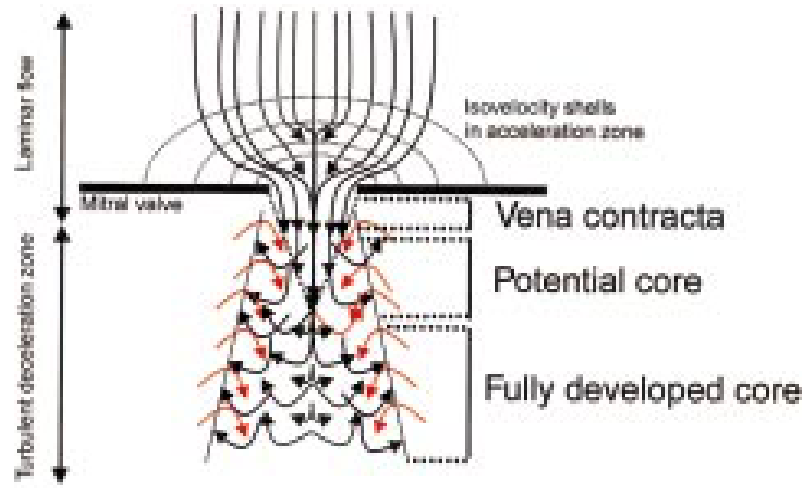

Figure 1 Flow patterns associated with regurgitation through a restrictive orifice. 
on quantification of the laminar flow in the acceleration zone or vena contracta. Methods to quantify this laminar flow are unsuitable for mild regurgitation (measurement of the proximal isovelocity surface area) or are currently employed only as research tools (digital analysis of the convergence zone or the use of power Doppler to quantify the vena contracta ${ }^{12}$ ). As outlined above, increases in the driving pressure increase the magnitude of regurgitation to the first power; however, this is compounded by the fact that the jet spray area visualised by colour Doppler increases to the second power making the appearance of the regurgitant jet particularly sensitive to changes in blood pressure. Thirdly, variations in detection rates may be attributable to changes in machine settings relating to processing of the Doppler signal or to variability between individual manufacturers. This is a subject that has received relatively little attention in the literature and would be expected to be a particular problem for relatively subtle reverse flow signals unaccompanied by turbulence. In one of the few available studies, Jones and colleagues found that for the same degree of regurgitant flow, visualised jet area increased as a function of instrument gain and transmit frequency, while decreasing with increases in pulse repetition frequency. ${ }^{17}$ In addition, changes in clutter filter settings (often linked inversely related to gain), transducer frequency, and pulse train packet size all determine the magnitude of the visualised jet spray. ${ }^{11}$ Subsequent in vitro studies have suggested that, in addition to the effect of a variety of colour maps, differences between individual manufacturers' machines are attributable to differences in the autocorrelation velocity estimation algorithm used to encode variance. ${ }^{18}$

\section{Practical problems in using valvar regurgitation to predict clinical outcomes in adults}

The two papers ${ }^{12}$ in this issue of Heart relate to children and adolescents; in these populations the frequency of valvar regurgitation is low $(<3 \%)^{19} 20$ and its presence more likely to be pathologically significant. The problem in adults is that the frequency of mild mitral regurgitation increases with age $(19 \%$ by a mean age of 54 years in the Framingham cohort, ${ }^{21}$ exceeding $80 \%$ by 80 years $^{22}$ ) to an extent that makes it difficult to attribute much significance to its presence. Matters are further compounded by the poorer imaging windows often available in these patients.

Thus the conceptual process involved in attempting to relate pathological events to the presence of mild regurgitant flows on colour Doppler is far from straightforward. Current Doppler techniques visualise the velocity of turbulent jet sprays, not the regurgitant volume, and are heavily pressure dependent. In future, techniques utilising digital Doppler quantification or power analysis of the acceleration zone and vena contracta may provide more quantitative solutions to this problem. In the meantime any study attempting to quantify these events requires rigorous standardisation of Doppler settings, a restriction of the types of machines employed, and simultaneous measurement of blood pressure. Although the presence of mild regurgitation through apparently structurally normal valves may provide clinically useful information in paediatric populations, its high frequency of occurrence renders it of less practical use in adults.

CRISPIN DAVIES DAVID SAHN ${ }^{\star}$

Division of Cardiololgy,

${ }^{\star}$ Clinical Care Center for Congenital Heart Disease,

Oregon Health Sciences University,

3181 SW Sam Fackson Park Road,

Portland, 97219-3098,

Oregon, USA

Daviesc@ohsu.edu

1 Allen J, Thompson JDR, Lewis I, et al. Mitral regurgitation after anthracycline treatment for childhood malignancy. Heart 2001;85:430-2.

2 Figueroa FE, Fernandez MS, Valdes P, et al. Prospective comparison of clinical and echocardiographic diagnosis of rheumatic carditis: long term follow up of patients with subclinical disease. Heart 2001;85:407-10.

3 Schiller NB. Fen/phen and valvular heart disease: if it sounds too bad to be true, perhaps it isn't. 7 Am Coll Cardiol 1999;34:1159-62.

4 Barzilai B, Gessler CJ, Perez JE, et al. Significance of Doppler-detected mitral regurgitation in acute myocardial infarction. Am f Cardiol 1988;61: 220-3.

5 Otsuji Y, Handschumacher MD, Kisanuki A, et al. Functional mitral regurgitation. Cardiologia 1998;43:1011-16.

6 Hung J, Otsuji Y, Handschumacher MD, et al. Mechanism of dynamic regurgitant orifice area variation in functional mitral regurgitation: physiologic insights from the proximal flow convergence technique. $f \mathrm{Am}$ Coll Cardiol 1999;33:538-45.

$7 \mathrm{He}$ S, Fontaine AA, Schwammenthal E, et al. Integrated mechanism for functional mitral regurgitation: leaflet restriction versus coapting force: in vitro studies. Circulation 1997;96:1826-34.

8 Otsuji Y, Handschumacher MD, Schwammenthal E, et al. Insights from three-dimensional echocardiography into the mechanism of functional mitral regurgitation: direct in vivo demonstration of altered leaflet tethering mitral regurgitation: direct in vivo demonst

9 Yoganathan AP, Cape EG, Sung HW, et al. Review of hydrodynamic principles for the cardiologist: applications to the study of blood flow and jets by imaging techniques. F Am Coll Cardiol 1988;12:1344-53.

10 Grimes RY, Hopmeyer J, Cape EG, et al. How sensitive are jet centerline velocities to an opposing flow? Implications for using the centerline method to quantify regurgitant jet flow. F Biomech 1996;29:967-71.

11 Sahn DJ. Instrumentation and physical factors related to visualization of stenotic and regurgitant jets by Doppler color flow mapping. $F$ Am Coll Cardiol 1988;12:1354-65.

12 Buck T, Mucci RA, Guerrero JL, et al. The power-velocity integral at the vena contracta, a new method for direct quantitfication of regurgitant flow volume. Circulation 2000;102:1053-61.

13 Mori Y, Shiota T, Jones M, et al. Three-dimensional reconstruction of the color Doppler-imaged vena contracta for quantifying aortic regurgitation: studies in a chronic animal model. Circulation 1999;99:1611-17.

14 Kececioglu-Draelos Z, Goldberg SJ, Areias J, et al. Verification and clinical demonstration of the echo Doppler series effect and vortex shed distance. Circulation 1981;63:1422-8.

15 Simpson IA, Valdes-Cruz LM, Sahn DJ, et al. Doppler color flow mapping of simulated in vitro regurgitant jets: evaluation of the effects of orifice size and hemodynamic variables. F Am Coll Cardiol 1989;13:1195-207.

16 Thomas JD, Liu CM, Flachskampf FA, et al. Quantification of jet flow by momentum analysis. An in vitro color Doppler flow study. Circulation $1990 ; 81: 247-59$

17 Jones M, Hoit B, Eidbo E, et al. Variability of color flow imaging of regurgitant jets in an animal model of mitral insuficiency [abstract]. $\mathcal{F} \mathrm{Am}$ regurgitant jets in an animal
Coll Cardiol 1987;9:64A.

18 Tamura T, Elias W, Gunday E, et al. In vitro studies of variance (turbulence) indicators in color flow mapping systems: quantitative computer analysis of RGB digitised flow map images [abstract]. Circulation 1987;76 IV:141.

19 Thomson JD, Allen J, Gibbs JL. Left sided valvar regurgitation in normal children and adolescents. Heart 2000;83:185-7.

20 Brand A, Dollberg S, Keren A. The prevalence of valvular regurgitation in children with structurally normal hearts: a color Doppler echocardiographic study. Am Heart $\mathcal{f} 1992 ; 123: 177-80$

21 Singh JP, Evans JC, Levy D, et al. Prevalence and clinical determinants of mitral, tricuspid, and aortic regurgitation (the Framingham heart study) [published erratum appears in Am f Cardiol 1999;84:1143]. Am f Cardiol 1999;83:897-902.

22 Akasaka T, Yoshikawa J, Yoshida K, et al. Age-related valvular regurgitation: a study by pulsed Doppler echocardiography. Circulation 1987;76:262-5. 